\title{
ON THE COBLE QUARTIC AND FOURIER-JACOBI EXPANSION OF THETA RELATIONS
}

\author{
FRANCESCO DALLA PIAZZA AND RICCARDO SALVATI MANNI
}

\begin{abstract}
In [RSSS12], the authors conjectured equations for the universal Kummer variety in genus 3 case. Though, most of these equations are obtained from the Fourier-Jacobi expansion of relations among theta constants in genus 4, the more prominent one, Coble's quartic, cf. [Cob29] was obtained differently, cf. [GSM2] too.

The aim of the current paper is to show that Coble's quartic can be obtained as Fourier-Jacobi expansion of a relation among theta-constants in genus 4 . We get also one more relation that could be in the ideal described in [RSSS12].
\end{abstract}

\section{INTRODUCTION}

Let $\mathcal{X}_{g}(2,4)$ be the universal Kummer variety with a suitable level structure.

We are interested in the map

$$
\kappa_{g}: \mathcal{X}_{g}(2,4) \rightarrow \mathbf{P}^{2^{g}-1} \times \mathbf{P}^{2^{g}-1}
$$

given by

$$
(\tau, z) \mapsto(\ldots, \Theta[\varepsilon](\tau), \ldots) \times(\ldots, \Theta[\varepsilon](\tau, z), \ldots) .
$$

By basic facts about theta functions we know that this map is well defined and generically injective. We would like to discuss equations for the image.

First of all we have to recall that the projections on the two factors of the product define the maps

$$
\Theta_{g}: \mathcal{A}_{g}(2,4) \rightarrow \mathbf{P}^{2^{g}-1}, \quad \text { given by } \tau \mapsto(\ldots, \Theta[\varepsilon](\tau), \ldots)
$$

and

$$
\psi_{g}: K_{\tau}:=X_{\tau} / \pm 1 \rightarrow \mathbf{P}^{2^{g}-1}, \quad \text { given by } \tau \mapsto(\ldots, \Theta[\varepsilon](\tau, z), \ldots) .
$$

Research of the authors is supported in part by Cofin 2011. 
The first map is generically injective, the second is finite of order $2^{k-1}$ with $k$ equal to the number of irreducible blocks of the class of the period matrix $\tau$. In the irreducible case we have that the second map is an immersion, cf. [K91] and it is known that the ideal of the image is generated by forms of degree at most 4, cf. [TIR13]. So we can have equations for the Kummer variety. Quartic equations are of special interest. In genus 2 case there is only one quartic equation. It has been determined in several different way, cf, [KH93], [RSSS12] and [GSM2]. In particular, in [RSSS12] one method is described, explained by Grushevsky and the second author, that uses Fourier-Jacobi expansion of the unique relation among second order theta constants of genus 3 .

In genus 3 case the situation is more involved. In fact there is a peculiar equation: Coble's quartic in second order theta functions. This has the property that if $\tau$ is the period matrix of the jacobian variety of a smooth plane quartic, equivalently theta constants of the first order do not vanish at $\tau$, then the singular locus of the Coble's quartic is scheme theoretic isomorphic to the Kummer variety, cf. [LZ96]. In the two recent papers [RSSS12], [GSM2] has been given an explicit equation for Coble's quartic. The method used was restriction of a suitable equation to the boundary components of the Satake compactification of $\overline{\mathcal{A}}_{3}(2,4)$ in [RSSS12] and the link between plane quartics and projective invariants of seven points of $\mathbf{P}^{2}$ in [GSM2].

More quartic equations are discussed in [RSSS12]. All of them are obtained from Fourier-Jacobi expansion of relations in genus 4 obtained turning into relations between $\Theta[\alpha](Z)$ the Riemann relations. The aim of the current paper is to get Coble's quartic and possibly other relevant quartics as Fourier-Jacobi expansion of suitable relations among $\Theta[\alpha](Z)$ of genus 4 .

\section{ACKNOWLEDGEMENTS}

The authors thanks Bert van Geemen, Samuel Grushevsky, Manabu Oura, Cris Poor and David Yuen for interesting conversations. They are grateful to Samuel Grushevsky for his help on a first version of this manuscript.

\section{NotATIONS AND DEFINITIONS}

We denote by $\mathbb{H}_{g}$ the Siegel upper half-space of symmetric complex matrices with positive-definite imaginary part. An element $\tau \in \mathbb{H}_{g}$ is called a period matrix, and defines the complex abelian variety $X_{\tau}:=$ $\mathbb{C}^{g} / \mathbb{Z}^{g}+\tau \mathbb{Z}^{g}$. The group $\Gamma_{g}:=\operatorname{Sp}(2 g, \mathbb{Z})$ acts on $\mathbb{H}_{g}$ by automorphisms: 


$$
\begin{aligned}
\text { for } M:=\left(\begin{array}{ll}
a & b \\
c & d
\end{array}\right) \in \operatorname{Sp}(2 g, \mathbb{Z}) \text { the action is } \\
\qquad M \cdot \tau:=(a \tau+b)(c \tau+d)^{-1} .
\end{aligned}
$$

A period matrix $\tau$ is called reducible if there exists $M \in \Gamma_{g}$ such that

$$
M \cdot \tau=\left(\begin{array}{cc}
\tau_{1} & 0 \\
0 & \tau_{2}
\end{array}\right), \quad \tau_{i} \in \mathbb{H}_{g_{i}}, g_{1}+g_{2}=g
$$

otherwise we say that $\tau$ is irreducible. The quotient of $\mathbb{H}_{g}$ by the action of the symplectic group is $\mathcal{A}_{g}$, the moduli space of principally polarized abelian varieties (ppavs). This quotient is an analytic variety with finite quotient singularities, and by a well-known result of Satake also a quasi-projective variety. Indeed, it is the coarse moduli space associated to the moduli stack of ppav.

We consider the group $\Gamma_{g} \ltimes \mathbb{Z}^{2 g}$ where the semi-direct product is given by the natural action of $\Gamma_{g}$ on vectors of length $2 \mathrm{~g}$. This group acts on $\mathbb{H}_{g} \times \mathbb{C}_{g}$ by

$$
\left(M,\left(\begin{array}{c}
m \\
n
\end{array}\right)\right) \cdot(\tau, z)=\left(M \cdot \tau,(z+\tau m+n)(c \tau+d)^{-1}\right) .
$$

We would like to say that the quotient

$$
\mathcal{X}_{g}=\Gamma_{g} \ltimes \mathbb{Z}^{2 g} / \mathbb{H}_{g} \times \mathbb{C}^{g}
$$

is the universal abelian variety over $\mathcal{A}_{g}$. This is true in the sense of stacks, but not for coarse moduli spaces, in fact we have that in particular $\left(-1_{2 g},\left(\begin{array}{l}0 \\ 0\end{array}\right)\right)$ acts on each fibre by the involution $z \mapsto-z$. Hence we get the universal Kummer variety. It appears canonically in the toroidal compactifications of $\mathcal{A}_{g+1}$. In fact for any toroidal compactification the first boundary component is isomorphic to $\mathcal{X}_{g}$. Hence a method to study the universal Kummer variety of genus $g$ leads to consider (partial) toroidal compactifications of $\mathcal{A}_{g+1}$. We would like to use theta functions to parametrize such varieties. Hence it is rather natural to introduce some level structures that allow us to avoid the stabilizers and to use theta functions.

We define the subgroups of the symplectic group to be

$$
\begin{gathered}
\Gamma_{g}[n]:=\left\{M=\left(\begin{array}{ll}
a & b \\
c & d
\end{array}\right) \in \Gamma_{g} \mid M \equiv\left(\begin{array}{ll}
1 & 0 \\
0 & 1
\end{array}\right) \bmod n\right\}, \\
\Gamma_{g}[n, 2 n]:=\left\{M \in \Gamma_{g}(n) \mid \operatorname{diag}\left(a^{t} b\right) \equiv \operatorname{diag}\left(c^{t} d\right) \equiv 0 \bmod 2 n\right\} .
\end{gathered}
$$


The corresponding level moduli spaces of ppavs and level moduli spaces of ppavs with theta structure are denoted $\mathcal{A}_{g}(n)$ and $\mathcal{A}_{g}(n, 2 n)$, respectively.

In the case of the $\mathcal{A}_{g}(2,4)$ the stabilizer group is $\pm 1_{2 g}$ if and only if the corresponding period matrix is irreducible. We set $\mathcal{X}_{g}(2,4)$ the corresponding quotient for the action of the group

$$
G_{g}(2,4)=\Gamma_{g}[2,4] \ltimes 2 \mathbb{Z}^{2 g} .
$$

\section{THETA FUNCTIONS}

For a period matrix $\tau \in \mathbb{H}_{g}$ the principal polarization $\Theta_{\tau}$ on the abelian variety $A_{\tau}:=\mathbb{C}^{g} /\left(\mathbb{Z}^{g}+\tau \mathbb{Z}^{g}\right)$ is induced by the divisor of the theta function

$$
\theta(\tau, z):=\sum_{n \in \mathbb{Z}^{g}} \exp \left(\pi i\left({ }^{t} n \tau n+2^{t} n z\right)\right) .
$$

Notice that for fixed $\tau$ theta is a function of $z \in \mathbb{C}^{g}$, and its automorphy properties under the lattice $\tau \mathbb{Z}^{g}+\mathbb{Z}^{g}$ define the bundle $\Theta_{\tau}$.

Given a point of order two on $A_{\tau}$, which can be uniquely represented as $\frac{\tau \varepsilon+\delta}{2}$ for $\varepsilon, \delta \in \mathbb{F}_{2}^{g}$ (where $\mathbb{F}_{2}=\{0,1\}$ is the additive group), the first order theta function with characteristic $m=[\varepsilon, \delta]$ is

$$
\theta\left[\begin{array}{l}
\varepsilon \\
\delta
\end{array}\right](\tau, z):=\sum_{m \in \mathbb{Z}^{g}} \exp \pi i\left({ }^{t}\left(m+\frac{\varepsilon}{2}\right) \tau\left(m+\frac{\varepsilon}{2}\right)+2^{t}\left(m+\frac{\varepsilon}{2}\right)\left(z+\frac{\delta}{2}\right)\right) .
$$

We shall use the notation $\theta_{m}(\tau, z)$ when the characteristic will be clear from the context. A characteristic $m=[\varepsilon, \delta]$ is called even or odd depending on whether the scalar product $\langle\varepsilon, \delta\rangle \in \mathbb{F}_{2}$ is zero or one. The number of even (resp. odd) characteristics is $2^{g-1}\left(2^{g}+1\right)$ (resp. $\left.2^{g-1}\left(2^{g}-1\right)\right)$. As a function of $z$, a theta function is even or odd according to its characteristic. For $\varepsilon \in \mathbb{F}_{2}^{g}$ the second order theta function with characteristic $\varepsilon$ is

$$
\Theta[\varepsilon](\tau, z):=\theta\left[\begin{array}{l}
\varepsilon \\
0
\end{array}\right](2 \tau, 2 z)
$$

These functions are even and they are a basis of $H^{0}\left(A_{\tau}, 2 \Theta_{\tau}\right)$. In general under the map $z \rightarrow-z$ the space $H^{0}\left(A_{\tau}, n \Theta_{\tau}\right)$, for $n \geq 3$, splits in two subspaces, $H^{0}\left(A_{\tau}, n \Theta_{\tau}\right)^{ \pm}$, of dimension $\frac{n^{g} \pm 2^{g}}{2}$ and $\frac{n^{g} \pm 1}{2}$ according to the case $n$ even or odd respectively.

Since we shall use theta functions in genus $g$ and $g+1$ we will try to reserve the notations $\delta, \varepsilon, \sigma$ for the genus $g$ case and $\alpha, \beta$ or $[*, \varepsilon]$ for the genus $g+1$ case. 
The square of any theta function with characteristic is a section of $2 \Theta_{\tau}$, and the basis for the space of sections of this bundle is given by theta functions of the second order. Riemann's addition formula is an explicit expression for the product of two theta functions with characteristics (cf. [I], Theorem 2, p. 139). In particular:

$$
\theta\left[\begin{array}{l}
\varepsilon \\
\delta
\end{array}\right](\tau, z)^{2}=\sum_{\sigma \in \mathbb{F}_{2}^{g}}(-1)^{\delta \cdot \sigma} \Theta[\sigma](\tau, 0) \Theta[\sigma+\varepsilon](\tau, z) .
$$

Moreover we have

$$
\Theta[\delta](\tau, z) \Theta[\delta+\varepsilon](\tau, z)=\frac{1}{2^{g}} \sum_{\sigma \in \mathbb{F}_{2}^{g}}(-1)^{\delta \cdot \sigma} \theta\left[\begin{array}{l}
\varepsilon \\
\sigma
\end{array}\right](\tau, 2 z) \theta\left[\begin{array}{l}
\varepsilon \\
\sigma
\end{array}\right](\tau, 0),
$$

which is valid for all $\tau, z$ and $\delta, \varepsilon$. Similarly we have also:

$$
\theta\left[\begin{array}{l}
\varepsilon \\
\delta
\end{array}\right](\tau, 2 z) \theta\left[\begin{array}{l}
\varepsilon \\
\delta
\end{array}\right](\tau, 0)=\sum_{\sigma \in \mathbb{F}_{2}^{g}}(-1)^{\delta \cdot \sigma} \Theta[\sigma](\tau, z) \Theta[\sigma+\varepsilon](\tau, z) .
$$

We recall a result about theta functions that is consequence of Riemann's relations. In [GSM1] the following result has been proved

Lemma 1. We have the following identity:

$$
\sum_{\varepsilon, \delta \in \mathbb{F}_{2}^{g}} \theta^{8}\left[\begin{array}{l}
\varepsilon \\
\delta
\end{array}\right](\tau, z)=\sum_{\varepsilon, \delta \in \mathbb{F}_{2}^{g}} \theta^{6}\left[\begin{array}{l}
\varepsilon \\
\delta
\end{array}\right](\tau, 0) \theta^{2}\left[\begin{array}{l}
\varepsilon \\
\delta
\end{array}\right](\tau, 2 z)
$$

Theta constants are restrictions of theta functions to $z=0$; we drop the argument $z=0$ in the notations for theta constants. All theta constants with odd characteristics vanish identically in $\tau$, while theta constants with even characteristics and all theta constants of the second order do not vanish identically.

\section{Modular forms AND Codes}

Here we recall the notion of multiplier system and modular form, see $[\mathrm{F}]$ for details. Let $\Gamma \subset \Gamma_{g}$ a congruence subgroup, a map $v: \Gamma \rightarrow \mathbb{C}$ is called a multiplier system of weight $r / 2, r \in \mathbb{Z}$ if $v(M)^{l}=1$ for all $M \in \Gamma$ and for some $l \in \mathbb{N}$ and defining:

$$
j_{r}(M, \tau):=v(M) \operatorname{det}(c \tau+d)^{r / 2}, \quad M=\left(\begin{array}{ll}
a & b \\
c & d
\end{array}\right) \in \Gamma, \quad \tau \in \mathbb{H}_{n}
$$

it satisfies the two conditions $j_{r}\left(M_{1} M_{2}, \tau\right)=j_{r}\left(M_{1}, M_{2} \tau\right) j_{r}\left(M_{2}, \tau\right)$, i.e. $j_{r}$ is a cocycle, and $j_{r}\left(-1_{2 g}, \tau\right)=1$, if $-1_{2 g} \in \Gamma$. If $r$ is even there are no ambiguity in the choice of the square root and the multiplier system is a character of $\Gamma$. Then a modular form of weight $r / 2$ with respect 
to the multiplier system $v$ is a holomorphic function $f: \mathbb{H}_{n} \rightarrow \mathbb{C}$ with the following properties:

a. $f(M \cdot \tau)=v(M) \operatorname{det}(c \tau+d)^{r / 2} f(\tau)$ for all $M \in \Gamma$;

b. for every $M \in \Gamma$ the function:

$$
\left(\left.f\right|_{r / 2} M\right)(\tau):=\operatorname{det}(c \tau+d)^{-r / 2} f(M \tau)=v(M) f(\tau)
$$

is bounded in domains of the kind $y \geq y_{0}>0$ with $\tau=x+i y$ and $y_{0}$ arbitrary.

We denote $[\Gamma, r / 2, v]$ the vector space of such modular forms. We shall consider the graded ring

$$
A(\Gamma, v):=\bigoplus_{k=0}^{\infty}\left[\Gamma, k / 2, v^{k}\right]
$$

We omit the multiplier if it is trivial.

All theta constants with characteristics are modular forms of weight one half and suitable multiplier with respect to $\Gamma(4,8) \subset \operatorname{Sp}(2 g, \mathbb{Z})$, while all theta constants of the second order are modular forms of weight one half and a different multiplier $\chi$ with respect to $\Gamma(2,4)$. This last case can be formalized as follows: there is a theta map

$$
\Theta_{2}: \mathbb{C}\left[F_{\varepsilon}: \varepsilon \in \mathbb{F}_{2}^{g}\right] \rightarrow A\left(\Gamma_{g}[2,4], \chi\right)
$$

sending $F_{\varepsilon}$ to $\Theta[\varepsilon]$. We refer to [Run93] for the details on the theta map.

We know that the group $\Gamma_{g}$ is generated by the elements $J=\left(\begin{array}{cc}0 & -1 \\ 1 & 0\end{array}\right)$ and $t(S)=\left(\begin{array}{ll}1 & S \\ 0 & 1\end{array}\right)$ for integral symmetric $S$.

Moreover it acts on the second order theta-constants. We recall the action of the generators, cf. [Run93] for details. We denote by

$$
\vec{\Theta}_{2}=(\ldots, \Theta[\varepsilon], \ldots)
$$

the vector of second order theta constants. Thus we have

$$
\left.\vec{\Theta}_{2}\right|_{\frac{1}{2}} t(S)=D_{S} \vec{\Theta}_{2} \text { and }\left.\vec{\Theta}_{2}\right|_{\frac{1}{2}} J=T_{g} \vec{\Theta}_{2},
$$

where

$$
\left.D_{S}=\operatorname{diag}\left(i^{t} a S a\right)\right)_{a \in \mathbb{F}_{2}^{g}} \text { and } T_{g}=\left(\frac{1+i}{2}\right)^{g}\left((-1)^{\langle a, b\rangle}\right)_{a, b \in \mathbb{F}_{2}^{g}} .
$$

The group

$$
H_{g}=\left\langle T_{g}, D_{S} \text { integral } \underset{6}{\operatorname{symmetric}} S\right\rangle \subseteq \mathrm{GL}\left(2^{g}, \mathbb{C}\right)
$$


is of finite order and

$$
H_{g} / \pm 1 \simeq \Gamma_{g} / \Gamma_{g}[2,4]^{*} .
$$

Here $\Gamma_{g}[2,4]^{*}$ is the subgroup of $\Gamma_{g}[2,4]$ defined by the condition $\operatorname{Tr}(a) \equiv g \bmod 4$. The map $\Theta_{2}$ results to be $\Gamma_{g} / \Gamma_{g}[2,4]^{*}$ equivariant. When $g$ is odd $\Gamma_{g}[2,4]$ is the extension of $\Gamma_{g}[2,4]^{*}$ by $-1_{2 g}$ that acts trivially.

We see that an $H_{g}$-invariant polynomial goes to a level one Siegel modular form of even weight under the map $\Theta_{2}$. We denote by $R_{g}$ the $H_{g}$-invariant subring of the homogeneous polynomials of even degree in $\mathbb{C}\left[F_{\alpha}: \alpha \in \mathbb{F}_{2}^{g}\right]$ and $R_{g}^{m}$ the vector space of $H_{g}$-invariant homogeneous polynomials of degree $m$, thus we have a theta map

$$
\theta_{2}: R_{g} \rightarrow A\left(\Gamma_{g}\right)
$$

whose image is contained in $A\left(\Gamma_{g}\right)^{(2)}$, i.e., in the subring of modular forms of even weight. The map is surjective when $g \leq 3$, cf. [Run93] and $[\mathrm{OSM}]$.

In genera 1,2 , the map is injective too. When $g=3$, it factorizes on a relation of degree 16: the Schottky relation that can be easily expressed in terms of first order theta constants, i.e.

$$
S(\tau)=\left(\frac{1}{8} \sum_{\text {meven }} \theta_{m}^{16}-\left(\frac{1}{8} \sum_{\text {meven }} \theta_{m}^{8}\right)^{2}\right) .
$$

Obviously considering subgroups of $H_{g}$ we get maps to ring of modular forms relative to groups $\Gamma$ sitting between $\Gamma_{g}[2,4]^{*}$ and $\Gamma_{g}$.

For our purposes we need to introduce a space of modular forms relative to a subgroup of the modular group containing $\Gamma_{g}[2,4]$. We set

$$
\Gamma_{g, 0}[2]:=\left\{M=\left(\begin{array}{ll}
a & b \\
c & d
\end{array}\right) \in \Gamma_{g} \mid c \equiv 0 \bmod 2\right\} .
$$

To this group, according to [RU95], corresponds the subgrop $H_{g, 4}$ that is generated by the all $D_{S}$ and

$$
A G L\left(g, \mathbb{F}_{2}\right):=\mathbb{F}_{2}^{g} \ltimes G L\left(g, \mathbb{F}_{2}\right),
$$

i.e the affine group of $G L\left(g, \mathbb{F}_{2}\right)$. It acts on $\mathbb{F}_{2}^{g}$ via

$$
(\varepsilon, M)(\delta)=M \delta+\varepsilon .
$$

This action can be naturally applied to second order theta constant, permuting them.

We describe the polynomials invariant with respect to the action of $H_{g, 4}$. We have that a monomial in the second order theta constant is admissible if it is invariant under the subgroup generated by the $D_{S}$. 
This is equivalent to the fact that the characteristic appearing satisfy some congruences, we refer to [RU95] for details.

Thus the invariants are obtained considering polynomials obtained summing on the $A G L\left(g, \mathbb{F}_{2}\right)$ orbits of admissible monomials. An invariant polynomial determines a string of $2^{g}$ number that are the multiplicity of the second order theta constants appearing in an admissible monomial occurring in the polynomial itself. For our case we need the following

\section{Lemma 2.}

$$
\operatorname{dim}\left[\Gamma_{3,0}[2], 6\right]=6 .
$$

A basis is given by the modular forms that are polynomials in the second order theta constants, related to the following admissible monomials

$$
\begin{gathered}
(12,0,0,0,0,0,0,0),(8,4,0,0,0,0,0,0),(4,4,4,0,0,0,0,0), \\
(6,2,2,2,0,0,0,0),(4,0,0,0,2,2,2,2),(5,1,1,1,1,1,1,1) .
\end{gathered}
$$

Proof. The dimension of the space is given in [RU95]. The above monomials have distinct orbits and they are obviouly independent, since, in genus 3 , the unique relation between theta constants of the second order has degree 16 .

Now we describe modular forms $F(Z)$ relative to $\Gamma_{g}$ that are polynomials in the $\Theta[\varepsilon]$. We assume that four divides the weight $k$. If this is the case we know that such modular forms are related to the weight enumerators $W_{C}^{g}$ of Type II code $C \subset \mathbb{F}_{2}^{2 k}$. A Type II code means a binary self-dual doubly-even code. To a weight enumerator is associated a theta series

$$
\theta_{\Lambda(C)}(Z)=\sum_{\lambda \in \Lambda(C)^{g+1}} \exp \left(2 \pi i \operatorname{tr}\left({ }^{t} \lambda Z \lambda\right)\right)
$$

for the lattice

$$
\Lambda(C)=\left\{\frac{1}{\sqrt{2}} x \in \mathbb{Z}^{2 k} / x \bmod 2 \in C\right\} .
$$

Let $F(\ldots, \Theta[\alpha](Z, 0), \ldots)$ be as above, it is a modular form for the full modular group, then, from [SM89], we know that

$$
F(Z)=\sum_{C} a_{C} \theta_{\Lambda(C)}(Z) .
$$

As example we recall that

$$
S(\tau)=\theta_{\Lambda\left(C_{1}\right)}(\tau)-\theta_{\Lambda\left(C_{2}\right)}(\tau) .
$$

Here $C_{1}$ and $C_{2}$ are the two classes of Type II code in degree 16 . 


\section{Fourier-JACOBI EXPANSION}

We briefly recall Fourier-Jacobi expansion of Siegel modular forms. To have it in genus $g$, we need to use modular forms of genus $g+1$. Let $f(Z)$ be a modular form of weight $r$ with $2 r \in \mathbb{N}$ and level $l$ with trivial multiplier. We decompose the variable $Z$ in blocks,

$$
Z=\left(\begin{array}{cc}
w & t^{t} \\
z & \tau
\end{array}\right), \text { with } \tau \in \mathbb{H}_{g}, z \in \mathbb{C}^{g}, w \in \mathbb{H}_{1},
$$

then, we have

$$
f(Z)=\sum_{n \in 2 \mathbb{N}} \phi_{n}(\tau, z) e^{\pi i n w / l} .
$$

In the case of theta constants we have, see [vG84]:

$$
\theta\left[\begin{array}{cc}
0 & \varepsilon \\
\delta_{1} & \delta
\end{array}\right]\left(\left(\begin{array}{cc}
w & t_{z} \\
z & \tau
\end{array}\right)\right)=\theta\left[\begin{array}{l}
\varepsilon \\
\delta
\end{array}\right](\tau, 0)+2 e^{\pi i \delta_{1}} q^{4} \theta\left[\begin{array}{l}
\varepsilon \\
\delta
\end{array}\right](\tau, z)+O\left(q^{16}\right)
$$

and

$$
\theta\left[\begin{array}{cc}
1 & \varepsilon \\
\delta_{1} & \delta
\end{array}\right]\left(\left(\begin{array}{cc}
w & { }^{t} z \\
z & \tau
\end{array}\right)\right)=2 e^{\pi i \delta_{1} / 2} q \theta\left[\begin{array}{l}
\varepsilon \\
\delta
\end{array}\right](\tau, z / 2)+O\left(q^{9}\right),
$$

where we let $q:=\exp (\pi i w / 4)$.

Similarly we have

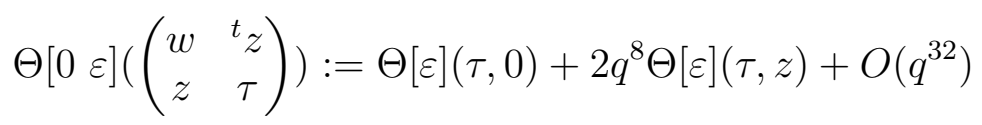

and

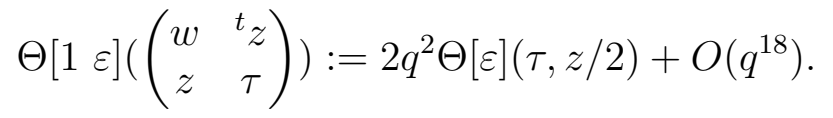

Thus, if $F_{g+1}$ is a modular form of genus $g+1$ that is a homogeneous polynomial of degree $2 r$ in the $\Theta[\alpha](Z)$, hence a modular form relative to the group $\Gamma_{g+1}[2,4]$, its $q$-expansion is of the form

$$
F_{g+1}(Z)=F^{0}(\tau)+F^{2}(\tau, z) q^{2}+\cdots+F^{2 n}(\tau, z) q^{2 n}+\ldots
$$

If $F_{g+1}$ is a modular form relative to larger group, some terms of the Fourier-Jacobi expansion vanish. If the group is $\Gamma_{g+1}$, we have

$$
F_{g+1}(Z)=F^{0}(\tau)+F^{8}(\tau, z) q^{8}+\cdots+F^{8 n}(\tau, z) q^{8 n}+\ldots
$$

When the modular group is $\Gamma_{g+1}[2]$ we get terms of the form $F^{4 n}(\tau, z)$. From now on we assume that $F_{g+1}$ is a modular form relative to $\Gamma_{g+1}$. 
We want to compute the first terms of the $q$-expansion of $F_{g+1}$. It is clear that $F^{0}(\tau)$ is the image of $F_{g+1}$ under the Siegel $\Phi$ operator, i.e.

$$
F^{0}(\tau)=\Phi\left(F_{g+1}\right)(\tau)=\lim _{q \rightarrow 0} F_{g+1}\left(\begin{array}{cc}
w & { }^{t} z \\
z & \tau
\end{array}\right) .
$$

For any $\tau \in \mathbb{H}_{g}, F^{8}(\tau, 2 z)$ is a section in $H^{0}\left(A_{\tau}, 8 \Theta\right)^{+}$. Hence we have

Lemma 3. Let $F_{g+1}(Z)$, be a modular form of weight $k$ and $\tau$ be a period matrix such that $\theta\left[\begin{array}{l}\varepsilon \\ \delta\end{array}\right](\tau) \neq 0$ for all even characteristics, then $F^{8 n}(\tau, 2 z)$ is a polynomial of degree $4 n$ in the $\Theta[\varepsilon](\tau, z)$. The coefficients are meromorphic modular forms of weight $k-2 n$.

Proof. It is an immediate consequence of the fact that in this case the Kummer variety $K_{\tau}$ is normally generated by $2 \Theta_{\tau}$. Hence the map

$$
\operatorname{Symm}^{4 n}\left(H^{0}\left(X_{\tau}, 2 \Theta_{\tau}\right)\right) \rightarrow H^{0}\left(X_{\tau}, 8 n \Theta_{\tau}\right)^{+}
$$

is surjective and we get for fixed $\tau F^{8 n}(\tau, 2 z)$ as a polynomial of degree $4 n$ in the $\Theta[\varepsilon](\tau, z)$. Considering $\tau$ as a variable, the modularity of $F_{g+1}(Z)$ implies the modularity of the coefficients.

To get an explicit expression for $F^{8}(\tau, 2 z)$ we need a further reordering of the monomials appearing in expressing the modular form $F_{g+1}(Z)$ as a sum of admissible monomials. We have

$$
F_{g+1}(Z)=f_{0}(Z)+f_{4}(Z)+f_{8}(Z)+\ldots
$$

Here $f_{i}$ is the sum of all monomials in the theta constants of the second order with the number 1 occurring exactly $i$ times as first entry in the characteristics.

We observe that there is a bijective map sending a polynomial in the $2^{g+1}$ variables $\Theta[\alpha](Z)$,

$$
p(\Theta[0, \delta](Z), \Theta[1, \varepsilon](Z))
$$

to the polynomial in the $2^{g+1}$ variables $\Theta[\delta](\tau), \Theta[\varepsilon](\tau, z)$,

$$
p(\Theta[\delta](\tau), \Theta[\varepsilon](\tau, z)) .
$$

With this notation we have the following

Proposition 4. Let $F_{g+1}$ be a modular form of weight $k$ that is a homogeneous polynomial in the $\Theta[\alpha](Z)$, then $F^{8}(\tau, 2 z)$ is of the form

$$
F^{8}(\tau, 2 z)=2^{4} f_{4}(\ldots, \Theta[\delta](\tau), \ldots, \Theta[\varepsilon](\tau, z))+H_{1}(\tau, z),
$$

with

$$
H_{1}(\tau, z)=\sum_{\varepsilon \in \mathbb{F}_{2}^{g}} \frac{\partial F^{0}}{\partial \Theta[\varepsilon]} \Theta[\varepsilon](\tau, 2 z) .
$$


Proof. We want to find the coefficient of $q^{8}$ in the Fourier-Jacobi expansion and this can be obtained as an immediate consequence of the Fourier-Jacobi expansion of the second order theta-constants (cf. §5). Thus, it is clear that we have to consider terms with quartic polynomials in the $\Theta[\varepsilon](\tau, z / 2)$ and terms that are linear in the $\Theta[\varepsilon](\tau, z)$. These appear only in the Fourier-Jacobi expansion of $f_{0}$. An easy computation gives $H_{1}(\tau, z)$.

We are interested in modular forms of this type vanishing along $\mathbb{H}_{4}$. Really, we restrict our attention to modular forms of weight 16 . This case has been studied in detail in [OPY]. It is known that there are 85 weight enumerators $W_{C}^{g+1}$ of degree 32 . In genus 4 case they span a 19 dimensional space. An explicit basis is given in [OPY]. They are the code polynomials. We denote by $\mathcal{C}$ the set of related codes. So from $[\mathrm{OPY}]$, we learned that the associated theta series span a space of dimension 14, hence we have five independent relations among them, denoted $\tilde{R}_{i}$, that can be expressed in terms of the basis of 19 weight enumerators chosen in $[\mathrm{OPY}]$ (see also $[\mathrm{O}]$ ) as:

$$
\begin{aligned}
\tilde{R}_{1}= & -3 \mathcal{C}_{2}+153 \mathcal{C}_{6}-420 \mathcal{C}_{10}+640 \mathcal{C}_{18}-83 \mathcal{C}_{24}-315 \mathcal{C}_{25}+28 \mathcal{C}_{27} \\
\tilde{R}_{2}= & -5 \mathcal{C}_{1}+110 \mathcal{C}_{2}-616 \mathcal{C}_{3}+880 \mathcal{C}_{4}+121 \mathcal{C}_{5}-121 \mathcal{C}_{6}-385 \mathcal{C}_{7}+16 \mathcal{C}_{23} \\
\tilde{R}_{3}= & -63 \mathcal{C}_{1}+990 \mathcal{C}_{2}-2016 \mathcal{C}_{3}-960 \mathcal{C}_{4}+957 \mathcal{C}_{5}-2610 \mathcal{C}_{6}+2520 \mathcal{C}_{7}+2520 \mathcal{C}_{10} \\
& +1280 \mathcal{C}_{18}-576 \mathcal{C}_{23}+498 \mathcal{C}_{24}-1890 \mathcal{C}_{25}+280 \mathcal{C}_{27}-1890 \mathcal{C}_{29}+960 \mathcal{C}_{67} \\
\tilde{R}_{4}= & -944 \mathcal{C}_{1}+11597 \mathcal{C}_{2}-22624 \mathcal{C}_{3}-6080 \mathcal{C}_{4}+1252 \mathcal{C}_{5}+3269 \mathcal{C}_{6}+23660 \mathcal{C}_{7} \\
& -10500 \mathcal{C}_{10}-4480 \mathcal{C}_{18}-5696 \mathcal{C}_{23}-1931 \mathcal{C}_{24}+7245 \mathcal{C}_{25}-1092 \mathcal{C}_{27} \\
& +22260 \mathcal{C}_{29}-37440 \mathcal{C}_{67}+21504 \mathcal{C}_{82} \\
\tilde{R}_{5}= & -64 \mathcal{C}_{1}-2041 \mathcal{C}_{2}+15400 \mathcal{C}_{3}-880 \mathcal{C}_{4}+14559 \mathcal{C}_{5}+42186 \mathcal{C}_{6}-38465 \mathcal{C}_{7} \\
& +50540 \mathcal{C}_{10}-117600 \mathcal{C}_{11}-225792 \mathcal{C}_{16}+3200 \mathcal{C}_{18}-26128 \mathcal{C}_{23}-63675 \mathcal{C}_{24} \\
& -21315 \mathcal{C}_{25}+16716 \mathcal{C}_{27}-47985 \mathcal{C}_{29}+409600 \mathcal{C}_{44}-29760 \mathcal{C}_{67}+21504 \mathcal{C}_{82},
\end{aligned}
$$

where the $\mathcal{C}_{i}$ are the elements of the basis of the weight enumerators.

Let $\tilde{R}(Z)$ be one of these relations

$$
\tilde{R}(Z)=\sum_{C \in \mathcal{C}_{32}} a_{C} \theta_{\Lambda(C)}(Z)=0,
$$

we consider in some detail its Fourier-Jacobi expansion. Referring to the notation of (2), we have

$$
\tilde{R}(Z)=r_{0}(\tau)+r_{1}(\tau, z) q^{8}+\ldots
$$


Now $r_{0}(\tau)$ is a modular form of weight 16 relative to $\Gamma_{3}$ vanishing identically on $\mathbb{H}_{3}$. So it is of the form

$$
r_{0}(\tau)=S(\tau)\left(a \frac{1}{8} \sum_{m \text { even }} \theta_{m}^{16}-b\left(\frac{1}{8} \sum_{\text {meven }} \theta_{m}^{8}\right)^{2}\right),
$$

with $a, b \in \mathbb{C}$.

Lemma 5. We can rewrite the relations $R_{1}, \ldots, R_{5}$ so that the corresponding $r_{0}(\tau)$ is equal to 0 in the first three cases, $S^{2}(\tau)$ in the fourth case and

$$
S(\tau)\left(\frac{1}{8} \sum_{m e v e n} \theta_{m}^{8}\right)^{2}
$$

in the last case.

Proof. We checked with a computer that all cases are possible and we got the relations in terms of the "old" relations. The following is a solution satisfying the above condition

$$
\begin{aligned}
& R_{1}=\tilde{R}_{3}-\frac{29481}{500158} \tilde{R}_{4}-\frac{2582}{2250711} \tilde{R}_{5} \\
& R_{2}=\tilde{R}_{2}+\frac{32395}{1000316} \tilde{R}_{4}-\frac{1474}{2250711} \tilde{R}_{5} \\
& R_{3}=\tilde{R}_{1}-\frac{2097}{250079} \tilde{R}_{4}-\frac{1996}{2250711} \tilde{R}_{5} \\
& R_{4}=\frac{7987}{8002528} \tilde{R}_{4}+\frac{77}{6001896 \tilde{R}_{5}} \\
& R_{5}=-\frac{1}{32} \tilde{R}_{1}=-W_{e_{8}} R_{0} .
\end{aligned}
$$

Here $R_{0}$ denotes a relation involving the weight enumerators of degree 24. This can be easily deduced from table 3 in [OPY], in fact the weight enumerators appearing in $\tilde{R}_{1}$ are products of the weight enumerator $W_{e_{8}}$ of degree 8 times weight enumerators of degree 24 . The relation $R_{0}$ is equivalent to that one that has been studied in [FO].

Proposition 6. In all these cases $r_{1}(\tau, z)$ is a quartic polynomial in the $\Theta[\varepsilon](\tau, z / 2)$ with holomorphic modular forms of weight 14 as coefficients.

Proof. We know that $r_{1}(\tau, z)$ has two summands. The first obviously gives the quartic polynomial and the second (that occurs only in the case of the fifth relation) we have to consider:

$$
\sum_{\varepsilon \in \mathbb{F}_{2}^{3}} \frac{\partial r_{0}(\tau)}{\partial \Theta[\varepsilon]} \Theta[\varepsilon](\tau, z)=\left(\frac{1}{8} \sum_{\text {meven }} \theta_{m}^{8}\right)^{2} \frac{\partial S(\tau)}{\partial \Theta[\varepsilon]} \Theta[\varepsilon](\tau, z)
$$


and by the chain rule:

$$
\sum_{\varepsilon \in \mathbb{F}_{2}^{3}} \frac{\partial r_{0}(\tau)}{\partial \Theta[\varepsilon]} \Theta[\varepsilon](\tau, z)=\left(\frac{1}{8} \sum_{m \text { even }} \theta_{m}^{8}\right)^{2} \sum_{m \text { even }} \frac{\partial S(\tau)}{\partial \theta_{m}^{2}} \theta_{m}(\tau, z)^{2} .
$$

Hence we need to compute

$$
\begin{aligned}
& \sum_{m \text { even }} \frac{\partial S(\tau)}{\partial \theta_{m}^{2}} \theta_{m}(\tau, z)^{2}=\sum_{m \text { even }}\left(\theta_{m}(\tau)^{14}-\left(\frac{1}{8} \sum_{n \text { even }} \theta_{n}(\tau)^{8}\right) \theta_{m}(\tau)^{6}\right) \theta_{m}(\tau, z)^{2} \\
& =\sum_{m \text { even }} \theta_{m}(\tau)^{14} \theta_{m}(\tau, z)^{2}-\frac{1}{8} \sum_{n \text { even }} \theta_{n}(\tau)^{8} \sum_{n \text { even }} \theta_{n}^{8}(\tau, z / 2) \\
& =G_{1}(\tau, z)+G_{2}(\tau, z) .
\end{aligned}
$$

Now the proposition follows from (1) and lemma 1.

For sake of clarity we write the Fourier-Jacobi expansion of the relations

$$
\begin{aligned}
R_{1} & =q^{8} r_{1,1}(\tau, z)+\ldots \\
R_{2} & =q^{8} r_{2,1}(\tau, z)+\ldots \\
R_{3} & =q^{8} r_{3,1}(\tau, z)+\ldots \\
R_{4} & =S^{2}(\tau)+q^{8} r_{4,1}(\tau, z)+\ldots \\
R_{5} & =S(\tau)\left(\frac{1}{8} \sum_{\text {meven }} \theta_{m}^{8}\right)^{2}+q^{8} r_{5,1}(\tau, z)+\ldots
\end{aligned}
$$

Since in the previous discussion we started with $\Gamma_{4}$-invariant relations we have that all $r_{i, 1}(\tau, 2 z)$ has the same structure as Coble's quartic, i.e. it is invariant under translation with points of order two of the abelian variety, hence each of them is of the form

$$
r_{i, 1}(\tau, 2 z)=s_{i, 1} Q_{1}+\ldots+s_{i, 15} Q_{15}, \quad i=1, \ldots, 5
$$

with

$$
\begin{aligned}
Q_{1}:=x_{000}^{4}+x_{001}^{4}+x_{010}^{4}+x_{100}^{4}+x_{110}^{4}+x_{101}^{4}+x_{011}^{4}+x_{111}^{4}, \\
Q_{2}:=x_{000}^{2} x_{001}^{2}+x_{010}^{2} x_{011}^{2}+x_{100}^{2} x_{101}^{2}+x_{110}^{2} x_{111}^{2}, \\
Q_{3}:=x_{000}^{2} x_{010}^{2}+x_{001}^{2} x_{011}^{2}+x_{100}^{2} x_{110}^{2}+x_{101}^{2} x_{111}^{2}, \\
Q_{4}:=x_{000}^{2} x_{011}^{2}+x_{010}^{2} x_{001}^{2}+x_{100}^{2} x_{111}^{2}+x_{110}^{2} x_{101}^{2}, \\
Q_{5}:=x_{000}^{2} x_{100}^{2}+x_{010}^{2} x_{110}^{2}+x_{001}^{2} x_{101}^{2}+x_{011}^{2} x_{111}^{2}, \\
Q_{6}:=x_{000}^{2} x_{101}^{2}+x_{010}^{2} x_{111}^{2}+x_{100}^{2} x_{001}^{2}+x_{110}^{2} x_{011}^{2},
\end{aligned}
$$




$$
\begin{aligned}
Q_{7} & :=x_{000}^{2} x_{110}^{2}+x_{010}^{2} x_{100}^{2}+x_{101}^{2} x_{011}^{2}+x_{001}^{2} x_{111}^{2}, \\
Q_{8} & :=x_{000}^{2} x_{111}^{2}+x_{010}^{2} x_{101}^{2}+x_{100}^{2} x_{011}^{2}+x_{110}^{2} x_{001}^{2}, \\
Q_{9} & :=x_{000} x_{010} x_{100} x_{110}+x_{001} x_{011} x_{101} x_{111}, \\
Q_{10} & :=x_{000} x_{001} x_{100} x_{101}+x_{010} x_{011} x_{110} x_{111}, \\
Q_{11} & :=x_{000} x_{011} x_{100} x_{111}+x_{001} x_{010} x_{101} x_{110}, \\
Q_{12} & :=x_{000} x_{001} x_{010} x_{011}+x_{100} x_{101} x_{110} x_{111}, \\
Q_{13} & :=x_{000} x_{010} x_{101} x_{111}+x_{001} x_{011} x_{100} x_{110} \\
Q_{14} & :=x_{000} x_{001} x_{110} x_{111}+x_{010} x_{011} x_{100} x_{101}, \\
Q_{15} & :=x_{000} x_{011} x_{101} x_{110}+x_{001} x_{010} x_{100} x_{111}
\end{aligned}
$$

Where $x_{\varepsilon}$ stands for $\Theta[\varepsilon](\tau, z)$. As a consequence of [GSM2] and the results in [GSM1], we have that such quartics are completely determined by

$$
s_{1} \in\left[\Gamma_{3,0}[2], 14\right] .
$$

In fact the coefficients $s_{1}, \ldots, s_{15}$ span a 15 dimensional representation of $\Gamma_{3}$ that has only a one dimensional $\Gamma_{3,0}[2]$ - invariant space, spanned by $s_{1}$.

Now for each relation $R_{i}$ in which occur about 50 millions admissible monomials, cf. [OPY], we have an expression of the corresponding quartic $r_{i, 1}(\tau, z)$, in which occur about one million terms. We know that these quartics are determined by the corresponding $s_{i, 1}$ in which occur about 5 thousands admissible monomials, cf. [RSSS12]. Really, in [RSSS12] and [GSM1] the related coefficient $s_{1}$ of the Coble's quartic appears as a monomial of degree 28 in the first order theta-constant. We need a description of $s_{1}$ as a polynomial in $\Theta[\varepsilon]$. This is an immediate consequence of the discussion in [RSSS12].

In fact we have

$$
s_{1}=\prod_{\varepsilon \neq 0}\left(\prod_{\delta /\langle\delta, \varepsilon\rangle=0} \theta\left[\begin{array}{l}
0 \\
\delta
\end{array}\right](\tau)-\prod_{\alpha /\langle\alpha, \varepsilon\rangle=1} \theta\left[\begin{array}{l}
0 \\
\alpha
\end{array}\right](\tau)\right)
$$

and as remarked in $[\operatorname{RSSS} 12]$ this is a polynomial in the $\theta\left[\begin{array}{l}\varepsilon \\ \delta\end{array}\right]^{2}(\tau)$ and in

$$
\prod_{\delta} \theta\left[\begin{array}{l}
0 \\
\delta
\end{array}\right](\tau)
$$

Using Riemann's relations in genus three, we get that also this term is a polynomial in the $\theta\left[\begin{array}{l}\varepsilon \\ \delta\end{array}\right]^{2}(\tau)$, hence we get an expression of the form

$$
s_{1}=p_{28}(\ldots, \Theta[\varepsilon], \ldots)_{\varepsilon \in \mathbb{F}_{2}^{3}} \text {. }
$$


It will have 5360 terms.

Now to each relation $R_{i}, i=1, \ldots, 5$, we can associate a quartic

$$
s_{i, 1} Q_{1}+\ldots+s_{i, 15} Q_{15}
$$

and we have the following

Proposition 7. The modular form $s_{1}(\tau)$ is contained in the ideal $\mathcal{I} \subset$ $\mathbb{C}[\ldots, \Theta[\varepsilon], \ldots]_{\varepsilon \in \mathbb{F}_{2}^{3}}$ generated by $s_{1,1}(\tau), s_{2,1}(\tau), \ldots, s_{5,1}(\tau)$.

Proof. A proof is obtained using a computer. The expressions for the coefficients are very long, but the computation involved does not require much time, once a couple of facts are taken into account. First we work on the polynomial ring $\mathbb{C}\left[\ldots, X_{\varepsilon}, \ldots\right]_{\varepsilon \in \mathbb{F}_{2}^{3}}$. Second, let $F_{1}, \ldots, F_{6}$ be a basis of $\left[\Gamma_{3,0}[2], 6\right]$, then instead of checking if $s_{1} \in \mathcal{I}$, we checked that the associated polynomial $p_{28}\left(\ldots, X_{\varepsilon}, \ldots\right)_{\varepsilon \in \mathbb{F}_{2}^{3}}$ belongs to the vector space $W$ spanned by the polynomials associated to

$$
S F_{1}, S F_{2}, \ldots, S F_{6}, s_{1,1}, \ldots, s_{5,1} \text {. }
$$

We found also that a basis is given by the first 8 vectors and $s_{5,1}$. Moreover, modulo the ideal generated by $S$, i.e. in $\mathbb{C}[\ldots, \Theta[\varepsilon], \ldots]_{\varepsilon \in \mathbb{F}_{2}^{3}}$ we have

$$
s_{1}=-\left(\frac{3787811}{13821931447380} s_{1,1}+\frac{914993}{4344035597748} s_{2,1}\right) .
$$

These results can be transferred to quartics vanishing along the universal Kummer variety of genus 3 . To have a complete statement, let us recall recall that in $[\mathrm{OPY}]$, there is a distinguished relation

$$
\begin{aligned}
573102233555 \text { BigNorm } & =151595494160 \tilde{R}_{1}-292362643392 \tilde{R}_{2} \\
& +82765857152 \tilde{R}_{3}+5300722416 \tilde{R}_{4} \\
& +230972544 \tilde{R}_{5} .
\end{aligned}
$$

Hence we have

Proposition 8. From Fourier-Jacobi expansion of a relation in genus 4 among theta series related to even self dual codes of rank 32, one obtains 3 independent quartics vanishing along the universal Kummer variety of genus 3. A basis is given by Coble's quartic, the term in the expansion of Big Norm and the term in the expansion of $R_{5}$.

From the previous proposition we obtain 
Corollary 9. The genus 4 relation, whose $r_{1}(\tau, z)$ coefficient in the Fourier-Jacobi expansion is the Coble quartic, expressed in terms of the basis chosen in [OPY] for the 19 dimensional space of weight enumerators of degree 32 in genus 4 is:

$$
\begin{aligned}
\alpha R & =5765253288 \mathcal{C}_{1}-113833368957 \mathcal{C}_{2}+290742188352 \mathcal{C}_{3} \\
& +12522322560 \mathcal{C}_{4}-163886691540 \mathcal{C}_{5}+480649493775 \mathcal{C}_{6} \\
& -246978898320 \mathcal{C}_{7}-465679797660 \mathcal{C}_{10}-32350348800 \mathcal{C}_{11} \\
& -62112669696 \mathcal{C}_{16}-237874412160 \mathcal{C}_{18}+54434900352 \mathcal{C}_{23} \\
& -111473675885 \mathcal{C}_{24}+350248142475 \mathcal{C}_{25}-48264847708 \mathcal{C}_{27} \\
& +428125619460 \mathcal{C}_{29}+112676044800 \mathcal{C}_{44}-380665602240 \mathcal{C}_{67} \\
& +127956347904 \mathcal{C}_{82},
\end{aligned}
$$

with $\alpha=608164983684720$.

In [RSSS12] it is conjectured that the prime ideal of the universal Kummer variety in genus 3 is generated by 891 bihomogeneous polynomials in $(u, x)$ : the Schottky polynomial of degree $(16,0)$, the eight Coble derivatives of degree $(28,3)$, and the 882 polynomials of degree $(16,4)$ that comes from the Fourier-Jacobi expansion of relations among the second order theta constants induced by Riemann's relations of genus 4 .

Big Norm is the symmetrization of these relations, so its Fourier-Jacobi expansion fits in the conjectural description of the ideal of the universal Kummer variety. From our result, the same is true for Coble quartic. We still have the Fourier-Jacobi expansion of one more relation, e.g. $R_{5}$. In this case we have that the resulting polynomial of bidegree $(28,4)$ is reducible, since $R_{5}$ does it. The non vanishing factor, proportional to $\sum_{\text {meven }} \theta_{m}^{8}(\tau)$ has degree $(8,0)$, so we get a relation of degree $(20,4)$ related to the Fourier-Jacobi expansion of the relation of degree 24, i.e. $R_{0}$. This is not obviously contained in the ideal described in [RSSS12]. So we conclude raising the following question

Question 10. Is the quartic

$$
\frac{r_{5,1}(\tau, 2 z)}{\sum_{m \text { even }} \theta_{m}^{8}(\tau)}
$$

contained in the ideal described in [RSSS12]?

\section{REFERENCES}

[Cob29] A. Coble, Algebraic geometry and theta functions, volume 10 of American Mathematical Society Colloquium Publications, American Mathematical Society, Providence, R.I., (1929). 
[Dol12] I. Dolgachev, Classical algebraic geometry: a modern view, Cambridge University Press, Cambridge, (2012).

[F] E. Freitag, Complex Analysis 2: Riemann Surfaces, Several Complex Variables, Abelian Functions, Higher Modular Functions, Springer (2011).

[FO] E. Freitag and M. Oura, A theta relation in genus 4, Nagoya Math Jour 161, 69-83, (2001).

[vG84] B. van Geemen, Siegel modular forms vanishing on the moduli spaces of curves. Invent. Math., 78 :329-349, (1986).

[GSM1] S. Grushevsky and R. Salvati Manni, The superstring cosmological constant and the Schottky form in genus 5, Am. Jour.Math 133, Number 4, 1007-1027, (2011).

[GSM2] S. Grushevsky and R. Salvati Manni, On Coble's quartic, preprint arXiv:1212.1895.

[I] J. Igusa, Theta functions, Springer 1972.

[K91] G. Kempf, Complex Abelian Varieties and Theta Functions, In Universitext, 1991.

[KH93] A. Khaled, Equations des varietes de Kummer, Math. Ann. 295, 685701. (1993).

[LZ96] Y. Laszlo, Local structure of the moduli space of vector bundles over curves, Comment. Math. Helv. 71, 373-401, (1996).

[O] M. Oura, http://www.math.kochi-u.ac.jp/oura/index-e.html.

[OPY] M. Oura, C. Poor, D. Yuen, Toward the Siegel ring in genus 4, In International Journal Number Theory.

[OSM] M. Oura R Salvati Manni, On the image of code polynomials under theta map, JOURNAL OF MATHEMATICS OF KYOTO UNIVERSITY vol. 48, 895-906, (2008), preprint arXiv:0803.4389.

[RSSS12] Q. Ren, S. Sam, G. Schrader, and B. Sturmfels, The universal Kummer threefold, preprint arXiv:1208.1229.

[Run93] B. Runge, On Siegel modular forms. I, J. Reine Angew. Math., 436:5785, (1993).

[RU95] B. Runge, On Siegel modular forms. II, Nagoya Math. J, 138:179-197, (1995).

[SM89] R. Salvati Manni, Thetanullwerte and stable modular forms, Amer. J. Math., 111(3):435-455, (1989).

[TIR13] S. Tirabassi, Syzygies and equations of Kummer varieties Bull. London Math Soc, published online January 31, (2013).

Università "La Sapienza", Dipartimento di Matematica, Piazzale A. Moro 2, I-00185, Roma, Italy

E-mail address: dallapiazza@mat.uniroma1.it, f.dallapiazza@gmail.com

Università "La Sapienza", Dipartimento di Matematica, Piazzale A. Moro 2, I-00185, Roma, Italy

E-mail address: salvati@mat.uniroma1.it 